\title{
ON DUALITY OF EXPONENTIAL AND LINEAR FORGETTING
}

\author{
R. Kulhavý $\dot{y}^{* 1,2} \quad$ F. J. Kraus ${ }^{* *, 1}$ \\ ${ }^{*}$ Institute of Information Theory and Automation, Academy of Sciences of the Czech \\ Republic,CZ-182 08 Prague, Czech Republic \\ ** Automatic Control Laboratory, Swiss Federal Institute of Technology (ETH), CH-8092 \\ Zurich, Switzerland
}

\begin{abstract}
Regularized (stabilized) versions of exponential and linear forgetting in parameter tracking are shown to be dual to each other. Both are derived by solving essentially the same Bayesian decision-making problem where Kullback-Leibler divergence is used to measure (quasi)distance between posterior probability distributions of estimated parameters. The type of forgetting depends solely on the order of arguments in Kullback-Leibler divergence. This general view indicates under which conditions one technique is superior to the other. Applied to the case of ARX models, the approach results in a class of regularized (stabilized) forgetting strategies that are naturally robust with respect to poor system excitation.
\end{abstract}

Keywords: Parameter estimation, statistical inference, algorithms, non-stationary systems, adaptation, forgetting.

\section{INTRODUCTION}

Various techniques of forgetting of obsolete information can be used to make parameter estimation capable of tracking slow parameter changes. Adaptive properties of control and signal processing depends heavily on the performance of these techniques. It explains a steady interest in analysis of known and design of new forgetting strategies.

Forgetting is essentially a heuristic technique owing to the fact that no or just a vague model of parameter variations is typically considered in design of adaptive systems. Apart from windowing-estimation from a batch of recent data (Middleton et al., 1988), two basic techniques of forgetting are used in practice. One is exponential forgetting motivated by time-weighted (discounted) least squares (Jazwinski, 1970) or, more generally, by the idea of flattening

\footnotetext{
1 Partially supported by the Swiss National Fund under project 7 TRP JO 38631.

2 Partially supported by the Grant Agency of the Czech Republic under grant 102/94/0314.
}

likelihood or posterior density function (Peterka, 1981). The other technique is linear forgetting justified for ARX models by the Kalman filtering view of estimation of time-varying parameters. Parameter changes are described here through a state-space model-typically random walk with known covariance matrix of parameter increments (Ljung, 1987).

The loss of information caused by forgetting is known to be dangerous in the case of insufficient system excitation which is the rule rather than the exception in practice. In the case of ARX models, the loss of information can result in undesirable growth of some entries of the covariance matrix of regression coefficients. Different ways of regularizing or stabilizing the covariance update formula have been proposed, including regularized exponential forgetting (Kreisselmeier, 1990; Kulhavý and Zarrop, 1993) and stabilized linear forgetting (Milek and Kraus, 1991; Milek, 1995). The formal similarity of these two techniques is so striking that one asks if a deeper connection between both exists.

The present paper shows that exponential and linear forgetting can be seen as solutions to basically the same Bayesian 
decision-making problem, with only slightly different loss functions. A common framework obtained in this way is believed to give some hints as to under which conditions one technique should be preferred to the other.

\section{PROBLEM STATEMENT}

Consider a stochastic system on which data $d_{k}$ are observed at discrete time instants $k=1,2, \ldots$. A directly manipulated input $u_{k}$ and an indirectly affected output $y_{k}$ can be distinguished in the data, $d_{k}=\left(u_{k}, y_{k}\right)$. Let the sequence of data observed on the system up to time $t$ be denoted as $D_{t}=$ $\left(d_{1}, d_{2}, \ldots, d_{t}\right)$.

The dependence of the system output $y_{k}$ on previous data $u_{k}$, $D_{k-1}$ at $k=1, \ldots, t$ can be described quite generally by the conditional probability density function (p.d.f.)

$$
p\left(y_{k} \mid u_{k}, D_{k-1}, \theta\right)
$$

parametrized by a (possibly multivariate) parameter $\theta \in \Theta$.

General Bayesian Estimation. When the unknown parameter $\theta$ is interpreted as a random variable, the uncertainty of $\theta$ given the observed data $D_{t}$ is naturally described by the posterior p.d.f. $p\left(\theta \mid D_{t}\right)$ conditional on $D_{t}$. This is generally determined by Bayes' theorem. Provided the input generator employs no other information about $\theta$ than the observed data (see the natural conditions of control in Peterka (1981))

$$
p\left(u_{k} \mid D_{k-1}, \theta\right)=p\left(u_{k} \mid D_{k-1}\right), \quad k=1, \ldots, t,
$$

Bayes' rule simplifies to the formula

$$
p\left(\theta \mid D_{t}\right) \propto p(\theta) \prod_{k=1}^{t} p\left(y_{k} \mid u_{k}, D_{k-1}, \theta\right)
$$

where $\propto$ stands for proportionality, i.e., equality up to a normalizing factor.

Recursive Least Squares. An important special case of (1) arises when the output $y_{k}$ depends on previous data $u_{k}$, $D_{k-1}$ through a known finite-dimensional vector function $\phi\left(u_{k}, D_{k-1}\right)=\phi_{k}$ in the following special way

$$
y_{k}=\theta^{\prime} \phi_{k}+e_{k}, \quad e_{k} \sim N\left(0, \sigma^{2}\right) .
$$

Here $\theta^{\prime}$ denotes transposition of $\theta$. The dependence is thus linear in the unknown parameters. The stochastic component $e_{k}$ is supposed to be a discrete white noise, normally distributed with known (for simplicity) variance $\sigma^{2}$. The model (3) induces the conditional p.d.f. of the form

$$
p\left(y_{k} \mid \phi_{k}, \theta\right)=\frac{1}{\sqrt{2 \pi \sigma^{2}}} \exp \left(-\frac{1}{2 \sigma^{2}}\left(y_{k}-\theta^{\prime} \phi_{k}\right)^{2}\right) \text {. }
$$

Suppose the prior p.d.f. $p(\theta)$ is normal $N\left(\hat{\theta}_{0}, P_{0}\right)$. Then one can easily prove that the posterior p.d.f. $p\left(\theta \mid D_{t}\right)$ produced by Bayes' rule (2) is normal again $-N\left(\hat{\theta}_{t}, P_{t}\right)$ with datamodified statistics $\hat{\theta}_{t}$ and $P_{t}$. The mean $\hat{\theta}_{k}$ and covariance $P_{k}$ update recursively according to the formulae

$$
\begin{aligned}
\hat{\theta}_{k} & =\hat{\theta}_{k-1}+\frac{P_{k-1} \phi_{k}}{\sigma^{2}+\phi_{k}^{\prime} P_{k-1} \phi_{k}} \hat{e}_{k} \\
P_{k}^{-1} & =P_{k-1}^{-1}+\frac{\phi_{k} \phi_{k}^{\prime}}{\sigma^{2}}
\end{aligned}
$$

where

$$
\hat{e}_{k}=y_{k}-\hat{\theta}_{k-1}^{\prime} \phi_{k}
$$

stands for the prediction error. The recursive formulae (5)(6) coincide with the well-known algorithm of recursive least squares. Much of the literature on parameter tracking deals only with the model (3) (Ljung and Gunnarsson, 1990).

General Idea of Forgetting. The assumption of constant parameters $\theta$ in the model (1) or (3) is not realistic in typical applications and need to be released. One possibility is to consider an explicit model of parameter variations in estimation. Such a model is, however, rarely available in practice. What one can do instead is to modify directly the algorithm (2) or, in particular, (5)-(6) so to make it possible to track at least slow parameter changes. There are certainly many ways of doing that.

In the paper we describe a class of forgetting algorithms that are linked by the following common idea. Suppose that the parameters $\theta_{k}$ are time-variable. The uncertainty of $\theta_{k}$ given data $D_{k}$ observed up to time $k$ is described by the posterior p.d.f. $p\left(\theta_{k} \mid D_{k}\right)$. What can be said about the p.d.f. $p\left(\theta_{k+1} \mid D_{k}\right)$ of the parameters $\theta_{k+1}$ at the next time instant given the same data $D_{k}$ ?

Suppose that no explicit model of parameter changes is known. We quantify our prior information (and possibly information taken from data already available) by introducing an alternative p.d.f. $p^{*}\left(\theta_{k+1} \mid D_{k}\right)$. The problem is then to construct a p.d.f. $p\left(\theta_{k+1} \mid D_{k}\right)$ based on two hypotheses described by the posterior p.d.f. $p\left(\theta_{k} \mid D_{k}\right)$ (the case of no parameter changes) and the alternative p.d.f. $p^{*}\left(\theta_{k+1} \mid D_{k}\right)$ (the case of worst expected changes). For simplicity, in the following two sections, we use the notation $p_{0}(\theta), p_{1}(\theta)$ and $p^{*}(\theta)$ for the posterior, alternative and resulting p.d.f.'s, respectively.

\section{FORGETTING AS OPTIMIZATION PROBLEM}

We formulate the task of choosing $p^{*}$ given $p_{0}$ and $p_{1}$ as a Bayesian decision-making problem. In particular, we assign probabilities $\lambda$ and $1-\lambda$ to the hypotheses $p_{0}$ and $p_{1}$ and define the best decision $p^{*}$ as the p.d.f. minimizing expectation of a distance or divergence $d\left(p, p_{i}\right)$ between $p$ and $p_{i}, i=0,1$

$$
\min _{p}\left[\lambda d\left(p, p_{0}\right)+(1-\lambda) d\left(p, p_{1}\right)\right]
$$


The choice of a proper distance $d(\cdot, \cdot)$ is of fundamental importance. In the sequel, we consider Kullback-Leibler divergence (also known as relative entropy, informational divergence, information for discrimination)

$$
D(p \| q)=\int p(\theta) \log \frac{p(\theta)}{q(\theta)} \mathrm{d} \theta .
$$

It should be emphasized that Kullback-Leibler divergence is not a true distance since it is not symmetric and does not satisfy the triangle inequality. Other appealing properties make it, however, a favoured information measure in mathematical statistics and information theory (see Kullback (1959) or Cover and Thomas (1991); cf. Kulhavý (1995)).

The fact that Kullback-Leibler divergence is not symmetric in its arguments is crucial here. The general solution (8) can thus take on two forms which will be shown to be dual to each other.

Problem 1. Exponential Forgetting (EF):

$$
\min _{p}\left[\lambda D\left(p \| p_{0}\right)+(1-\lambda) D\left(p \| p_{1}\right)\right]
$$

Problem 2. Linear Forgetting (LF):

$$
\min _{p}\left[\lambda D\left(p_{0} \| p\right)+(1-\lambda) D\left(p_{1} \| p\right)\right]
$$

\subsection{General Solution}

To solve the above optimization problems, we need only the following property of Kullback-Leibler divergence.

Lemma 1. Kullback-Leibler divergence is always nonnegative, $D(p \| q) \geq 0$. Moreover, $D(p \| q)=0$ if and only if $p(\theta)=q(\theta)$ almost everywhere.

PROOF. See e.g. Cover and Thomas (1991).

Theorem 1. Suppose that the p.d.f.'s $p_{0}$ and $p_{1}$ are not mutually orthogonal, i.e., their product is not zero almost everywhere. Then the optimization problem EF (10) has a unique solution

$$
p^{*}(\theta) \propto p_{0}(\theta)^{\lambda} p_{1}(\theta)^{1-\lambda}
$$

PROOF. Let us denote $\lambda_{0}=\lambda$ and $\lambda_{1}=1-\lambda$. Then the function minimized in (10) can be rewritten as follows

$$
\begin{aligned}
& \sum_{i=0}^{1} \lambda_{i}\left(\int p(\theta) \log \frac{p(\theta)}{p_{i}(\theta)} \mathrm{d} \theta\right) \\
& =\int p(\theta) \log \frac{p(\theta)}{\frac{\prod_{i=0}^{1} p_{i}(\theta)^{\lambda_{i}}}{\int \prod_{i=0}^{1} p_{i}(\theta)^{\lambda_{i}} \mathrm{~d} \theta}} \mathrm{d} \theta \\
& \quad-\log \int \prod_{i=0}^{1} p_{i}(\theta)^{\lambda_{i}} \mathrm{~d} \theta \\
& =D\left(p \| p^{*}\right)+\text { const. }
\end{aligned}
$$

where $p^{*}$ is given by (12) and the constant is independent of $p$. The assumption that the p.d.f.'s $p_{0}$ and $p_{1}$ are not mutually orthogonal ensures $\int \prod_{i=0}^{1} p_{i}(\theta)^{\lambda_{i}} \mathrm{~d} \theta>0$. It follows by Lemma 1 that $p=p^{*}$ is the only minimizing solution of (10).

Theorem 2. Given any two p.d.f.'s $p_{0}$ and $p_{1}$, the optimization problem LF (11) has a unique solution

$$
p^{*}(\theta)=\lambda p_{0}(\theta)+(1-\lambda) p_{1}(\theta) .
$$

PROOF. Let us denote $\lambda_{0}=\lambda$ and $\lambda_{1}=1-\lambda$ again. The function minimized in (11) can be rewritten in the following manner

$$
\begin{aligned}
& \sum_{i=0}^{1} \lambda_{i}\left(\int p_{i}(\theta) \log \frac{p_{i}(\theta)}{p(\theta)} \mathrm{d} \theta\right) \\
& =\int\left(\sum_{i=0}^{1} \lambda_{i} p_{i}(\theta)\right) \log \frac{\left(\sum_{i=0}^{1} \lambda_{i} p_{i}(\theta)\right)}{p(\theta)} \mathrm{d} \theta \\
& \quad-\int\left(\sum_{i=0}^{1} \lambda_{i} p_{i}(\theta)\right) \log \left(\sum_{i=0}^{1} \lambda_{i} p_{i}(\theta)\right) \mathrm{d} \theta \\
& \quad+\sum_{i=0}^{1} \lambda_{i}\left(\int p_{i}(\theta) \log p_{i}(\theta) \mathrm{d} \theta\right) \\
& =D\left(p^{*} \| p\right)+\text { const. }
\end{aligned}
$$

where $p^{*}$ is given by (13) and the constant is independent of $p$. Once again, Lemma 1 implies that $p=p^{*}$ is the only minimizing solution of (11).

\subsection{Exponential Family}

The general solutions (12) and (13) are functional expressions that are difficult to implement if nothing more is said about the p.d.f.'s $p_{0}$ and $p_{1}$. In recursive estimation, it is natural to prefer models that admit a finite-dimensional sufficient statistic. Such models are known to result in posterior p.d.f.'s that belong to an exponential family. A general exponential family $\mathcal{E}$ is composed of p.d.f.'s of the following form

$$
p_{\beta}(\theta) \propto o(\theta) \exp \left(\beta^{\prime} b(\theta)\right)
$$

where $o(\theta)$ stands for the origin of the family, $b(\theta)$ is a vector of linearly independent basis functions $b_{i}(\theta)$ and $\beta$ is a vector parameter specifying a particular p.d.f. When $p_{0}$ and $p_{1}$ take the above form, the solutions to the optimization problems $\mathrm{EF}$ and LF become more tractable.

Theorem 3. If $p_{0}$ and $p_{1}$ are exponential p.d.f.'s (14) with parameters $\beta_{0}$ and $\beta_{1}$, respectively, then the solution $p^{*}$ to the problem $E F$ is an exponential p.d.f. (14) with the parameter

$$
\beta^{*}=\lambda \beta_{0}+(1-\lambda) \beta_{1}
$$


PROOF. Substituting (14) for $p^{*}, p_{0}$ and $p_{1}$ in (12), we have

$$
\beta^{* \prime} b(\theta)=\lambda \beta_{0}^{\prime} b(\theta)+(1-\lambda) \beta_{1}^{\prime} b(\theta) .
$$

The proposition follows by the fact that the basis functions $b_{i}$ are common to $p_{0}$ and $p_{1}$ and linearly independent.

It is easy to see that there is no closed-form solution to the problem LF in the exponential family. The mixture (13) of exponential p.d.f.'s does not belong to the exponential family any more. A natural way of simplifying (13) is to construct an exponential approximation of it

$$
\min _{p \in \mathcal{E}} D\left(p^{*} \| p\right), p^{*}(\boldsymbol{\theta})=\lambda p_{0}(\boldsymbol{\theta})+(1-\lambda) p_{1}(\boldsymbol{\theta}) .
$$

It turns out that this approximation has the same effect as restricting the solution of (11) right away to the exponential family $\mathcal{E}$.

Problem 3. Restricted Linear Forgetting (LFr):

$$
\min _{p \in \mathcal{E}}\left[\lambda D\left(p_{0} \| p\right)+(1-\lambda) D\left(p_{1} \| p\right)\right]
$$

Lemma 2. The solution of (17) coincides with the solution of (16).

PROOF. The proposition follows directly from the proof of Theorem 2 where the equality

$$
\lambda D\left(p_{0} \| p\right)+(1-\lambda) D\left(p_{1} \| p\right)=D\left(p^{*} \| p\right)+\text { const. }
$$

was shown to hold.

Theorem 4. If $p_{0}$ and $p_{1}$ are exponential p.d.f.'s (14) with parameters $\beta_{0}$ and $\beta_{1}$, respectively, then the solution to the problem (17) is an exponential p.d.f. $p_{\beta^{*}}$ (14) with the parameter $\beta^{*}$ such that the expectations $\hat{b}^{*}=E_{p_{\beta^{*}}}[b(\theta)], \hat{b}_{0}=$ $E_{p_{0}}[b(\theta)], \hat{b}_{1}=E_{p_{1}}[b(\theta)]$ of the basis vector function $b(\theta)$ with respect to the p.d.f.'s $p_{\beta^{*}}, p_{0}, p_{1}$, respectively, satisfy

$$
\hat{b}^{*}=\lambda \hat{b}_{0}+(1-\lambda) \hat{b}_{1}
$$

PROOF. Owing to Lemma 2, it is sufficient to find the projection of $p^{*}=\lambda p_{0}+(1-\lambda) p_{1}$ onto the exponential family $\mathcal{E}$ in the sense of (16). The necessary condition for $p_{\beta^{*}} \in \mathcal{E}$ to minimize $D\left(p^{*} \| p_{\beta}\right)$ is

$$
\nabla_{\beta} D\left(p^{*} \| p_{\beta^{*}}\right)=E_{p_{\beta^{*}}}[b(\theta)]-E_{p^{*}}[b(\theta)]=0 .
$$

It follows from (16) and linearity of the expectation operator that

$$
E_{p_{\beta^{*}}}[b(\theta)]=\lambda E_{p_{0}}[b(\theta)]+(1-\lambda) E_{p_{1}}[b(\theta)]
$$

which gives immediately (18).
The sufficiency of (18) follows by checking the matrix of second partial derivatives of $D\left(p^{*} \| p_{\beta}\right)$

$$
\nabla_{\beta}^{2} D\left(p^{*} \| p_{\beta^{*}}\right)=\operatorname{Cov}_{p_{\beta^{*}}}[b(\theta)] .
$$

The covariance matrix of $b(\theta)$ is positive definite unless $p_{\beta^{*}}$ is singular (a Dirac function). The latter case can happen only when $p_{0}$ and $p_{1}$ are singular and identical. But then $p_{\beta^{*}}=$ $p_{0}=p_{1}$ is the minimizing solution trivially.

The parameters $\beta$ and $\hat{b}$ are known as dual parametrizations of the exponential family. The symmetry of solutions (15) and (18) reveals clearly this duality.

\subsection{Normal Family}

A well-known special case of the exponential family is the normal family $\mathcal{N}$ composed of p.d.f.'s of the following form

$$
p_{\hat{\theta}, P}(\theta)=\frac{|P|^{-\frac{1}{2}}}{\sqrt{2 \pi}} \exp \left(-\frac{1}{2}(\theta-\hat{\theta})^{\prime} P^{-1}(\theta-\hat{\theta})\right) \text {. }
$$

Here $\hat{\theta}$ and $P$ denote the mean and covariance of a particular p.d.f. The basis functions $b_{i}(\theta)$ in (14) are composed of entries of the vector $\theta$ and the matrix $\theta \theta^{\prime}$. Provided that the p.d.f.'s $p_{0}$ and $p_{1}$ are normal (19), solutions to the optimization problems EF (10) and LFr (17) get very close to what is usually understood in the literature under names of exponential and linear forgetting, respectively.

Theorem 5. If $p_{0}$ and $p_{1}$ are normal (19) with parameters $\hat{\theta}_{0}, P_{0}$ and $\hat{\theta}_{1}, P_{1}$, respectively, then the solution $p^{*}$ to the problem (10) is also normal (19) with the parameters $\hat{\theta}^{*}, P^{*}$ determined by the formulae

$$
\begin{aligned}
P^{*-1} \hat{\theta}^{*} & =\lambda P_{0}^{-1} \hat{\theta}_{0}+(1-\lambda) P_{1}^{-1} \hat{\theta}_{1} \\
P^{*-1} & =\lambda P_{0}^{-1}+(1-\lambda) P_{1}^{-1}
\end{aligned}
$$

PROOF. The fact that given normal p.d.f.'s $p_{0}$ and $p_{1}$, the p.d.f. $p^{*}(12)$ is normal again is trivial. Substituting (19) for $p^{*}, p_{0}$ and $p_{1}$ in (12), we find that the quadratic forms in the exponentials are related as follows

$$
\begin{aligned}
\left(\theta-\hat{\theta}^{*}\right)^{\prime} P^{*-1}\left(\theta-\hat{\theta}^{*}\right) & =\lambda\left(\theta-\hat{\theta}_{0}\right)^{\prime} P_{0}^{-1}\left(\theta-\hat{\theta}_{0}\right) \\
& +(1-\lambda)\left(\theta-\hat{\theta}_{1}\right)^{\prime} P_{1}^{-1}\left(\theta-\hat{\theta}_{1}\right) .
\end{aligned}
$$

The recursion (20) follows after straightforward algebraic manipulations.

Theorem 6. Let $p_{0}$ and $p_{1}$ be normal (19) with parameters $\hat{\theta}_{0}, P_{0}$ and $\hat{\theta}_{1}, P_{1}$, respectively. Denote $\Delta \hat{\theta}=\hat{\theta}_{0}-\hat{\theta}_{1}$. Then the solution $p^{*}$ to the problem (17) is also normal (19) with the parameters $\hat{\theta}^{*}, P^{*}$ determined by the formulae 


$$
\begin{aligned}
\hat{\theta}^{*} & =\lambda \hat{\theta}_{0}+(1-\lambda) \hat{\theta}_{1} \\
P^{*} & =\lambda P_{0}+(1-\lambda) P_{1}+\lambda(1-\lambda) \Delta \hat{\theta} \Delta \hat{\theta}^{\prime}
\end{aligned}
$$

PROOF. We apply Theorem 4 to the normal family. Since the basis functions in this case are formed by the entries of the vector $\theta$ and the matrix $\theta \theta^{\prime}$, Theorem 4 yields the formulae

$$
\begin{aligned}
E_{p^{*}}[\theta] & =\lambda E_{p_{0}}[\theta]+(1-\lambda) E_{p_{1}}[\theta], \\
E_{p^{*}}\left[\theta \theta^{\prime}\right] & =\lambda E_{p_{0}}\left[\theta \theta^{\prime}\right]+(1-\lambda) E_{p_{1}}\left[\theta \theta^{\prime}\right] .
\end{aligned}
$$

The first equality gives directly (22). The formula (22) follows from the second equality after substitution $E_{p}\left[\theta \theta^{\prime}\right]=$ $P+\hat{\theta} \hat{\theta}^{\prime}$ and straightforward algebraic manipulations.

The formulae stated in Theorems 5 and 6 simplify considerably when the alternative mean $\hat{\theta}_{1}$ is set equal to the posterior mean $\hat{\theta}_{0}$.

Corollary 1. If $\hat{\theta}_{1}=\hat{\theta}_{0}$, then the recursion (20) simplifies to

$$
\begin{aligned}
\hat{\theta}^{*} & =\hat{\theta}_{0}, \\
P^{*-1} & =\lambda P_{0}^{-1}+(1-\lambda) P_{1}^{-1} .
\end{aligned}
$$

Corollary 2. If $\hat{\theta}_{1}=\hat{\theta}_{0}$, then the recursion (21) simplifies to

$$
\begin{aligned}
& \hat{\theta}^{*}=\hat{\theta}_{0}, \\
& P^{*}=\lambda P_{0}+(1-\lambda) P_{1} .
\end{aligned}
$$

Note that the recursions (22) and (23) essentially correspond to regularized exponential forgetting in Kulhavý and Zarrop (1993) and stabilized linear forgetting in Milek and Kraus (1991), respectively.

Note that results analogous to those obtained above could be derived using other paradigms of statistical decision-making such as minimum distance or min-max (barycentre) principles (Kulhavý and Zarrop, 1993).

\section{DUAL PROPERTIES}

This section shows that the general view of exponential and linear forgetting adopted in Sec. 3 provides more insight into the different effects of exponential and linear forgetting.

Duality of Kullback-Leibler divergence. Kullback-Leibler divergence $D\left(p_{A} \| p_{B}\right)$ is known to be sensitive to close-tozero values in the second argument $p_{B}$. Loosely speaking, the integral value of $D\left(p_{A} \| p_{B}\right)$ is small enough only when the regions of very small $p_{B}$-probability have also very small $p_{A}$-probability.
What does that mean in our case? In exponential forgetting we minimize a weighted arithmetic mean of $D\left(p \| p_{i}\right)$. Thus, $p^{*}(\theta)$ is almost zero for those parameter values $\theta$ for which either $p_{0}(\theta)$ or $p_{1}(\theta)$ is close to zero. As a result, $p^{*}$ is closer to that of the p.d.f.'s $p_{0}, p_{1}$ which is more concentrated, less flat. It is typically the posterior p.d.f. $p_{0}$.

In linear forgetting we minimize a weighted arithmetic mean of $D\left(p_{i} \| p\right)$. For this reason, $p^{*}(\theta)$ differs significantly from zero for those parameter values for which either $p_{0}(\theta)$ or $p_{1}(\theta)$ is large enough. Consequently, $p^{*}$ extends over both $p_{0}$ and $p_{1}$. The same holds even for restricted linear forgetting when the resulting p.d.f. $p^{*}$ is required to belong to an exponential family.

Geometric versus arithmetic mean. Looking at the resulting formulae (12) and (13) rather than the original optimization problems, we get another interpretation of exponential and linear forgetting. Exponential forgetting results in $p^{*}(\theta)$ which is, up to a normalizing constant, a weighted geometric mean of $p_{0}(\theta)$ and $p_{1}(\theta)$. Linear forgetting makes $p^{*}(\theta)$ a weighted arithmetic mean of $p_{0}(\theta)$ and $p_{1}(\theta)$. This reinforces the point made in the preceding paragraph. With exponential forgetting, $p^{*}(\theta)$ gets close to zero if either $p_{0}(\theta)$ or $p_{1}(\theta)$ is almost zero. With linear forgetting, $p^{*}(\theta)$ becomes significantly greater than zero if either $p_{0}(\theta)$ or $p_{1}(\theta)$ is large enough.

Harmonic versus arithmetic mean. Let us look now at the formulae (22) and (23) that are in the form closest to the standard notions of exponential and linear forgetting. Clearly, exponential forgetting makes the covariance matrix $P^{*}$ a weighted harmonic mean of $P_{0}$ and $P_{1}$ while linear forgetting results in $P^{*}$ which is a weighted arithmetic mean of $P_{0}$ and $P_{1}$. The following result shows that except trivial cases, linear forgetting produces a larger increase of covariance of $\theta$ than exponential forgetting.

Proposition 1. Assume that the covariance matrices $P_{0}$ and $P_{1}$ are both positive definite and that $\lambda \in(0,1)$. Then the difference of the covariance matrices $P_{\mathrm{EF}}^{*}=$ $\left(\lambda P_{0}^{-1}+(1-\lambda) P_{1}^{-1}\right)^{-1}$ and $P_{\mathrm{LF}}^{*}=\lambda P_{0}+(1-\lambda) P_{1}$

$$
\begin{aligned}
P_{\mathrm{LF}}^{*}-P_{\mathrm{EF}}^{*}=\lambda(1-\lambda) P_{\mathrm{EF}}^{*}\left(P_{0}^{-1}-P_{1}^{-1}\right)\left(\lambda P_{1}\right. \\
\left.+(1-\lambda) P_{0}\right)\left(P_{0}^{-1}-P_{1}^{-1}\right) P_{\mathrm{EF}}^{*}
\end{aligned}
$$

is a positive semidefinite matrix. If, in addition, $P_{1}>P_{0}$, the difference is a positive definite matrix.

PROOF. Applying straightforward algebraic manipulations we get

$$
\begin{aligned}
& \left(P_{\mathrm{EF}}^{*}\right)^{-1}\left(P_{\mathrm{LF}}^{*}-P_{\mathrm{EF}}^{*}\right)\left(P_{\mathrm{EF}}^{*}\right)^{-1} \\
& =\left(\left(P_{\mathrm{EF}}^{*}\right)^{-1} P_{\mathrm{LF}}^{*}-I\right)\left(P_{\mathrm{EF}}^{*}\right)^{-1}
\end{aligned}
$$




$$
\begin{aligned}
& =\lambda(1-\lambda)\left(P_{0}^{-1} P_{1}+P_{1}^{-1} P_{0}-2 I\right)\left(P_{\mathrm{EF}}^{*}\right)^{-1} \\
& =\lambda(1-\lambda)\left(P_{0}^{-1}-P_{1}^{-1}\right)\left(P_{1}-P_{0}\right)\left(P_{\mathrm{EF}}^{*}\right)^{-1} \\
& =\lambda(1-\lambda)\left(P_{0}^{-1}-P_{1}^{-1}\right)\left(\lambda P_{1}+(1-\lambda) P_{0}\right)\left(P_{0}^{-1}-P_{1}^{-1}\right)
\end{aligned}
$$

The proposition follows easily from the last expression.

The above property can be seen as a matrix generalization of the well-known fact that the weighted harmonic mean is bounded from above by the weighted arithmetic mean (de Finetti, 1974, Sec. 2.9).

Ellipsoid of concentration versus covariance ellipsoid. A different update of the covariance matrix $P$ due to exponential and linear forgetting can be well envisaged geometrically using one of the following notions. An ellipsoid of concentration

$$
\|\Delta \theta\|_{P^{-1}}^{2}=\Delta \theta^{\prime} P^{-1} \Delta \theta=\text { const. }
$$

is defined in the space of possible parameter deviations $\Delta \theta=$ $\theta-\hat{\theta}$ with metric given by the information matrix $P^{-1}$. A covariance ellipsoid

$$
\|\phi\|_{P}^{2}=\phi^{\prime} P \phi=\text { const. }
$$

is defined in the space of possible regressors $\phi$ with metric given by the covariance matrix $P$.

The ellipsoid of concentration is particularly useful for analysis of exponential forgetting (22) since

$$
\|\Delta \theta\|_{P^{*-1}}^{2}=\lambda\|\Delta \theta\|_{P_{0}^{-1}}^{2}+(1-\lambda)\|\Delta \theta\|_{P_{1}^{-1}}^{2} .
$$

On the contrary, the covariance ellipsoid is a more natural tool for visualizing linear forgetting (21) because

$$
\|\phi\|_{P^{*}}^{2}=\lambda\|\phi\|_{P_{0}}^{2}+(1-\lambda)\|\phi\|_{P_{1}}^{2} .
$$

\section{CONCLUDING REMARKS}

The paper presents a class of naturally regularized or stabilized forgetting strategies close to the standard techniques of exponential and linear forgetting. The general formulation of the forgetting task as a kind of Bayesian decision-making problem makes it possible to say more about the mutual relationship of exponential and linear forgetting.

To sum up, exponential forgetting turns out less sensitive to the choice of the alternative p.d.f. $p_{1}(\theta)$ or, specifically, the alternative mean $\hat{\theta}_{1}$ and covariance $P_{1}$ than linear forgetting. This makes it a suitable technique for the situations when knowledge of parameter variations is really vague and uncertain. The alternative hypothesis still may play an important role. Under poor system excitation, information about parameters in poorly excited directions of the parameter space becomes increasingly determined by the alternative (typically prior) knowledge.
Since linear forgetting puts more weight to the alternative hypothesis than exponential forgetting, it seems to be a better choice for the cases when a good knowledge of the underlying system is available. Such prior knowledge like information about different rates of parameter variations in different directions can be well used to shape the alternative covariance $P_{1}$. With linear forgetting the effect of this information on the parameter estimates is considerably stronger-even in well "excited" directions of the parameter space. As a result, the quality of parameter tracking can be substantially improved. The higher sensitivity of linear forgetting calls for more caution, however. If information supplied by the user does not agree with the actual parameter variations, parameter tracking may deteriorate drastically.

\section{REFERENCES}

Cover, T. M. and J. A. Thomas (1991). Elements of Information Theory. Wiley. New York.

de Finetti, B. (1974). Theory of Probability; A Critical Introductory Treatment. Vol. 1. Wiley. New York.

Jazwinski, A. H. (1970). Stochastic Processes and Filtering Theory. Academic Press. New York.

Kreisselmeier, G. (1990). Stabilized least-squares type adaptive identifiers. IEEE Trans. Automat. Control 35, 306310.

Kulhavý, R. (1995). A Kullback-Leibler distance approach to system identification. In: Preprints of the IFAC Symposium on Adaptive Systems in Control and Signal Processing. Budapest, Hungary. pp. 55-66.

Kulhavý, R. and M. B. Zarrop (1993). On a general concept of forgetting. Internat. J. Control 58(4), 905-924.

Kullback, S. (1959). Information Theory and Statistics. Wiley. New York.

Ljung, L. (1987). System Identification: Theory for the User. Prentice-Hall. Englewood Cliffs, N.J.

Ljung, L. and S. Gunnarsson (1990). Adaptation and tracking in system identification - a survey. Automatica - J. IFAC 26(1), 7-21.

Middleton, R. H., G. C. Goodwin, D. J. Hill and D. Q. Mayne (1988). Design issues in adaptive control. IEEE Trans. Automat. Control 33(1), 50-58.

Milek, J. J. (1995). Stabilized Adaptive Forgetting in Recursive Parameter Estimation. Dissertation ETH No. 10893. Swiss Federal Institute of Technology. Zurich.

Milek, J. J. and F. J. Kraus (1991). Stabilized least squares estimators for time variant processes. In: Proceedings of the 1st IFAC Symposium on Design Methods of Control Systems. Vol. 1. Zurich, Switzerland. pp. 430-435.

Peterka, V. (1981). Bayesian approach to system identification. In: Trends and Progress in System Identification (P. Eykhoff, Ed.). Chap. 8, pp. 239-304. Pergamon. Elmsford, N.Y. 